\title{
FLAT BEAM SPOT SIZES MEASUREMENT IN THE SLC-FINAL FOCUS*
}

\author{
P. Raimondi and F. J. Decker \\ Stanford Linear Accelerator Center, Stanford University, Stanford, CA 94309 USA
}

With the switch to flat beam operation in the SLC during the 1993 run [1], it has become necessary to develop an algorithm that is capable of measuring the beam spot sizes at the Final Focus Interaction Point (IP). This algorithm uses the correct beam-beam deflection formula for the more general flat-beam case, since the round beam approximation is no longer valid [2]. The application of this formula to the IP spot size measurements in the SLC Final Focus is the subject of this paper.

\section{DERIVATION OF THE BEAM-BEAM DEFLECTION FORMULA IN THE FLAT BEAM CASE}

The assumptions used in the theoretical evaluation of the beam-beam deflection are:

(a) Gaussian and upright distributed beams with sigmas in the three dimensions $\sigma_{\mathrm{Z}-}, \sigma_{\mathrm{X}-}, \sigma_{\mathrm{y}-}$ for electrons, and $\sigma_{\mathrm{z}+}, \sigma_{\mathrm{x}+}, \sigma_{\mathrm{y}+}$ for positrons

(b) $y=O$ (on plane colliding beams)

(c) $\alpha_{\max } \times \sigma_{z-}<2 \sigma_{x-}$ (no disruption )

It can be proved that the overall deflection angle is proportional to the electric field generated by the convolutions of the two beam space distributions [3]. Moreover, the Bassetti expression for the electric field $E(x)$ is valid for Gaussian distributed beam [4]. Hence, after some algebra, the total deflection angle $\alpha(x)$ in the $x$-plane of the $e^{-}$beam, due to the interaction with the $e^{+}$beam, as a function of their distance $x$ is:

It can be proved that the overall deflection angle is proportional to the electric field generated by the convolutions of the two beam space distributions [3]. Moreover, the Bassetti expression for the electric field $E(x)$ [4] is valid for the Gaussian distributed beam. Hence, after some algebra, the total deflection angle $\alpha(x)$ in the $x$-plane of the $\mathrm{e}^{-}$beam (due to the interaction with the $\mathrm{e}^{+}$beam) as a function of their distance $x$ is

$$
\begin{aligned}
\alpha(x)= & 2 \sqrt{\pi} \frac{N_{e+} r_{e}}{\gamma} \frac{x_{n} e^{x_{n}^{2}}}{x} \\
& \times\left[\operatorname{erfc}\left(\frac{\Sigma_{y}}{\Sigma_{x}} x_{n}\right)-\operatorname{erfc}\left(x_{n}\right)\right],
\end{aligned}
$$

*Work supported by DE-AC03-76SF00515. where

$$
\begin{gathered}
x_{n}=\frac{x}{\sqrt{2\left(\Sigma_{y}^{2}-\Sigma_{x}^{2}\right)}} \\
\operatorname{erfc}(x)=\frac{2}{\sqrt{\pi}} \int_{x}^{+\infty} d t e^{-t^{2}},
\end{gathered}
$$

$N_{e+}$ is the number of particles in the positron bunch $r_{e}$ is the classical electron radius

$\gamma$ is the beam energy over the electron mass, and

$$
\Sigma_{y}=\sqrt{\sigma_{y-}^{2}+\sigma_{y+}^{2}} \text {, and } \Sigma_{x}=\sqrt{\sigma_{x-}^{2}+\sigma_{x+}^{2}}
$$

with some further algebra, we can also express the deflection in an integral form simpler than the one used in [2]:

$$
\alpha(x)=\frac{N_{e+} r_{e}}{\gamma} \frac{x}{\Sigma_{x}^{2}} \int_{0}^{1} d t \frac{e^{-\left(x^{2} / 2 \Sigma_{x}^{2}\right) t}}{\sqrt{1+\left(a^{2}-1\right) t}},
$$

where

$$
a=\Sigma_{y} / \Sigma_{x}
$$

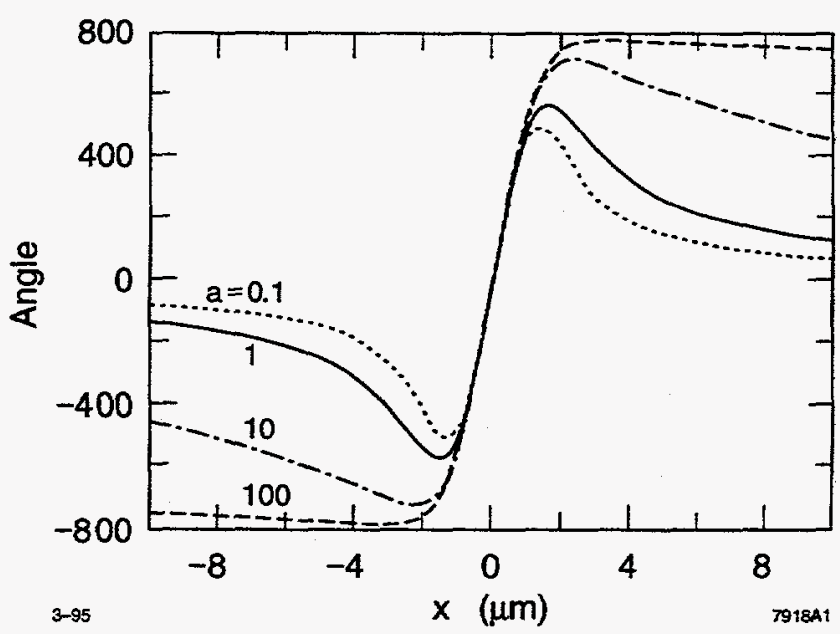

Figure 1. Beam-beam deflection at different aspect ratios (not in scale), $a=\Sigma_{y} / \Sigma_{x}$.

It can be shown that in the limit $\Sigma_{\mathrm{x}} \sim \Sigma_{\mathrm{y}}, \mathrm{Eq} . \sim(1)$ becomes the "round beam-beam deflection" expression [2], and in the limit $\Sigma_{\mathrm{y}} \gg \Sigma_{\mathrm{x}}$ it becomes the "error function" as expected in the infinite flatness case (see Fig. 1). This DISTRIBUTION OF THIS DOCUMENT IS UNLIMITED

Presented at the 16th IEEE Particle Accelerator Conference (PAC 95) and International Conference on High-Energy Accelerators, Dallas, Texas, May 1--5, 1995 
behavior is much more evident in the integral expressiomn for $\alpha(x)$, since in the case $\Sigma_{\mathrm{x}}=\Sigma_{\mathrm{y}}$, the integral is solvable in terms of elementary functions; whereas, in the case $\Sigma_{\mathrm{y}} \gg \Sigma_{\mathrm{x}}$, it is simply expressible as an error function.

\section{MEASUREMENT OF BEAM SPOT SIZES AT THE SLC INTERACTION POINT}

The typical beam sigmas at the SLC-IP are: $\sigma_{\mathrm{z}-}, \sigma_{\mathrm{z}+} \sim$ $600 \mu \mathrm{m}, \sigma_{\mathrm{x}-}, \sigma_{\mathrm{x}+}-2.5 \mu \mathrm{m}$, and $\sigma_{\mathrm{y}-}, \sigma_{\mathrm{y}+} \sim 0.8 \mu \mathrm{m}$. Furthermore, the maximum deflection angle $\alpha_{\max }$ is of the order of $300 \mu \mathrm{rad}$.

It can be shown that $\alpha(x)$ is finite and real for any $\Sigma_{\mathrm{X}}$ and $\Sigma_{\mathrm{y}}>0$.

Since conditions (b) and (c) are satisfied [condition (a) is assumed to be correct], Eq. (1) was applied to fit the beam-beam deflection in the SLC 1993 run [1]. It is possible to notice from (1) that the deflection in one plane is a function of the distance between the two beams, and of both $\Sigma_{\mathrm{X}}$ and $\Sigma_{\mathrm{y}}$, so that, in principle, the deflection in one plane is able to give information on both spot sizes. A further advantage of (1) is that the overall scale factor of the deflection angle is a known quantity, depending only by the other-beam charge (measured elsewhere); specifically:

$$
2 \sqrt{\pi} \frac{N_{e+} r_{e}}{\gamma}=1118.4 N_{e+},
$$

with the given SLC-beam energy ( $45.64 \mathrm{GeV}$ ), and expressing the deflection angle in $\mu \mathrm{rad}$, the sigmas in $\mu \mathrm{m}$ and the number of $e^{+}$in $10^{10}$ units.

The fitting of the beam-beam deflection therefore involves only four free parameters: the two beam spotsizes, the absolute distance between the two beams, and the overall offset of the measured deflection angle. The last two parameters are mainly caused by the residual offsets of the beam-position-monitor readings.

Figure 2 shows two typical beam-beam scans in the
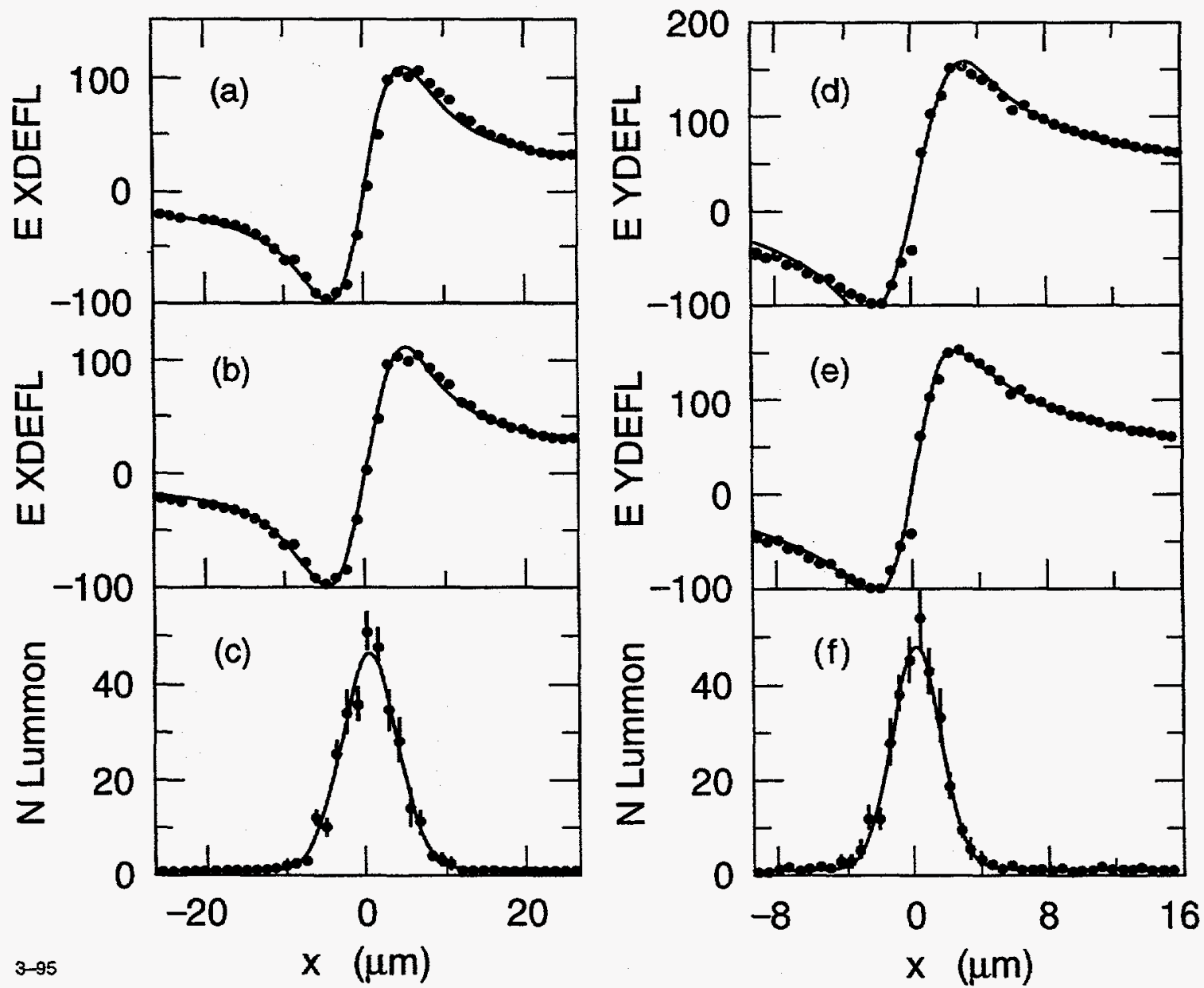

Figure 2. Beam-beam scan fits in a typical SLC running conditions: (a) fit of the $x$-deflection using the round beam-beam formula, (b) fit of the $x$-deflection using the flat beam-beam formula, (c) fit of $\Sigma_{x}$ using the Bhabha-counts, (d) fit of the y-deflection using the round beam-beam formula, (e) fit of the y-deflection using the flat beam-beam formula, ( $f$ ) it of $\Sigma_{y}$ using the Bhahba-counts; where $A=$ deflection offset, $B=$ scale factor, $\mathrm{C}=$ on plane beam spot size, $\mathrm{D}=$ position offset, $\mathrm{F}=$ other plane beam spot size. 


\section{DISCLAIMER}

Portions of this document may be illegible in electronic image products. Images are produced from the best available original document. 
two planes, fitted with the round-beam formula and with the flat formula. Also shown in Fig. 2 are the beam sigmas, as measured by using a signal proportional to the Bhabha scattering at low angle. The measured spot sizes using the two techniques are in good agreement.

The accuracy in determining both beam spot sizes with a single beam-beam scan is typically better than $5 \%$.

\section{CONCLUSIONS}

The use of the Eq. (1) to fit the beam-beam deflection at the SLC IP gives us a very accurate spot-size monitor to tune SLC Final Focus, and to optimize and estimate the SLC luminosity. The agreement of the data with the theory is excellent showing that the assumption (a) is also correct. Furthermore, the disruption effect can be estimated to be still small enough to affect the behavior of the beam-beam scans at the typical 1993 running condition, leading to anunderestimate of the real SLC luminosity of about $8 \%$, while it has been a more serious problem in the SLC 1994 where the $\Sigma_{y}$ was of the order of $0.9 \mu \mathrm{m}$ and condition (c) was no longer valid, causing an appreciable difference in the behavior of the measured beam-beam deflection with respect to (1). Corrections of the flat beam-beam formula (1) must be included to take this effect into account.

\section{REFERENCES}

[1] C. Adolphsen et al., "Flat beams in the SLC," PAC93.

[2] W. Koska et al., "Beam-Beam Deflection as a Beam Tuning Tool at the SLAC Linear Collider", SLACPUB-4919.

[3] V.Ziemann, "Beam beam deflection and signature curves for elliptic beams", SLAC-CN-382.

[4] M. Bassetti and G.A. Erskine, "Closed Expression for the Electrical field of a Two-Dimensional Gaussian Charge," CERN-ISR-TH/80-06.

[5] C. Pisin, "SLC Flat Beam Disruption Effects," SLAC Memorandum, April 1993. 